\title{
Optimizing the L3 handover delay in Small Objects Networks mobile IPv6 based
}

\author{
Ali EL KSIMI, Cherkaoui LEGHRIS \\ RTM Team \\ Hassan 2 university of Casablanca, \\ Faculty of Science and Technology \\ Mohammedia, Morocco
}

\begin{abstract}
The technologies evolution has made possible the emergence of new types of machines which allow their users to full move on different networks. In the same context, the arrival of Mobile IPv6 allows a mobile node to maintain a continuous connectivity. Handover management is one of its most critical processes. $\mathrm{L} 3$ handover is the most important because a major proportion of the total handover latency occurs in this stage. In this paper, we have proposed a new algorithm called FURA (Fast Unsolicited Router Advertisement) to optimize the L3 handover delay. Our algorithm is going to minimize the $\mathrm{L3}$ handover delay by optimizing Router Advertisement interval and avoiding Duplicate Address Detection process. Overall, this algorithm showed a significant effectiveness and might be used in a future networks investigations.
\end{abstract}

Keywords—Mobile IPv6;handover delay;CoA;Home Agent.

\section{INTRODUCTION}

SmObNet6 (Small Objects Network IPv6 based) is a generic term which is used to define either small or large networks that could interconnect several small objects. The use of IPv6 protocol in terms of communication, collecting and exchanging data between objects, represents a common point between these networks within the internet infrastructure.

In a mobile IPv6 [1], when a mobile node wants to move and attach to a new network, it needs to obtain a new IPv6 address to continue communications with its correspondent node. The IP routing mechanism relies on the information found in IP headers, so that, they can deliver data to the proper nodes, thus a movement from one location to another requires the old IP connections to be turn down and new connections to be established. Mobile IPv6 provides a solution to overcome this problem without major changes at the routers or nodes in a network.

In Mobile IPv6 protocol, each mobile node is identified by a set of IPv6 addresses [2]. When being in the home network, a Home Agent (HA) assigns a local address to the mobile node and it is always reachable via its Home Address (HoA). When the node is away from its home, it obtains a Care of Address (CoA) from the foreign router and registers this CoA with its HA.

The job of the HA is to intercept any packets destined for the mobile node while it is roaming in a foreign network and tunnel it to the mobile node. The inherent problem in this scenario is that, a timely configuration of $\mathrm{CoA}$ is required for continuous communication. The time taken for mobile node to obtain a new IPv6 address and register it with the HA is the overall handover delay.

The handover delay is the primary cause of packet loss in a network and it is found to be a bottleneck in performance studies conducted previously $[1,2]$. Proposed schemes to reduce handover delay operate either at the IP layer [3] or above it.

In this work, we propose an algorithm called FURA (Fast Unsolicited Router Advertisement) to reduce handover delay on IP layer.

The rest of this paper is organized as follows: In the next section, we present a related work to our field. In section 3, we study an overview of Mobile IPv6 while in section 4; we focus on IP delay issue and proposed solution. A conclusion and perspectives are provided in last section.

\section{RELATED WORK}

One of the important issues in wireless communications is handover delay. Because of this, many researches have tried to overcome with appropriate approach to reduce the associated time delay during the handover process.

In [4], the authors work specifically on the registration delay component. They make the assumption that the link layer delay can be considered equal to zero for link layer technologies supporting soft handover. They also consider the movement detection delay depends upon the frequency of router advertisement and could be large in a bandwidth constraint environment.

In [5], the authors have proposed a new method to improve F-PMIPv6 by controlling the handover management with optimized routing and propose an extension of PMIPv6 called improved PMIP.

In [6], the authors have proposed a novel approach to Reduce Handover delay in Proxy Mobile IPv6 Multi-Homing based. The proposed method, combined with Modified Mobility Access Gateway (M_MAG), handles the handover session dynamically.

In [7], the authors have proposed a fuzzy based model which reduces the delay and packet loss. 
In [8], the authors have presented a new control function called Extended Handover Control Function (E-HCF) in order to improve the handover performance in the context of Mobile IPv6 over wireless networks. They have focused on the handover performance at the Network Layer.

In [9], the authors have proposed the comparison of mechanisms for reducing Handover Latency and Packet Loss Problems of Route Optimization in MIPv6.

To more improve the handoff delay in layer 3 , we use in this paper, a new approach based on an algorithm called FURA (Fast Unsolicited Router Advertisement). Our numerical results validated by simulations show that the FURA algorithm enables to decrease the handover delay in Layer 3.

\section{MoBILE IPv6}

\section{A. Addressing IPv6}

MIPv6 is an IP-layer mobility management protocol for the IPv6 Internet. It allows a Mobile Node (MN) to transparently maintain connections while moving from one subnet to another. Each node is identified by its home address (HoA) although it may be connecting to through another network. When connecting through a foreign network, a mobile node sends its location information to a home agent (HA), which intercepts packets intended for the node and tunnels them to the current location. MIPv6 protocol allows mobile nodes to stay connected during the mobility whitin IPv6 internet. When $\mathrm{MN}$ moves from home network to a foreign network and wants to use their services, it sends a Router Solicitation (RS) message and in response to that, it get a Router Advertisement (RA) message. The MIPv6 architecture is shown in figure 1.

In MIPv6, the IPv6 home address (HA) is assigned to the mobile node. The mobile node obtains a new IPv6 address care-of-address (the CoA) on networks to which it connects.

The home agent accepts binding updates (BUs) from the mobile node informing the agent of the mobile node's location. The home agent then acts as proxy for the mobile node, intercepting traffic to the mobile node's home address and tunneling it to the mobile node.

The mobile node informs its home agent about its new address, and the correspondent node will be informed further. Because of the use of ingress filtering, the mobile node reverses tunnel return traffic to the home agent, so that the mobile node source address (that is, its home address) will always be topographically correct.

Mobile IPv6 gives ability to a mobile node to bypass the home agent when sending IP packets to a correspondent node. Optional extensions make direct routing possible in Mobile IPv6, though the extensions might not be implemented in all deployments of Mobile IPv6.

Direct routing is built into Mobile IPv6, and the direct routing function uses the IPv6 routing header and the IPv6 destination options header. The routing header is used for sending packets to the mobile node using its current $\mathrm{CoA}$, and the home address destination option is used to include the mobile node's home address, because the current CoA is the source address of the packet.

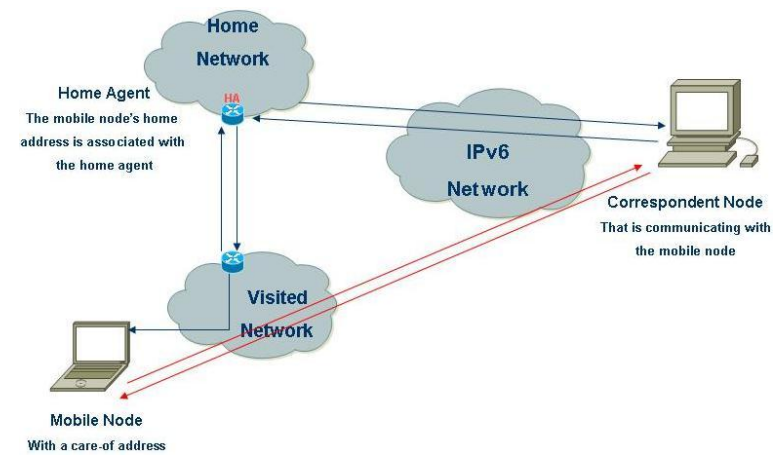

Fig.1.MIPv6 scenario

\section{B. Handover in MIPv6}

The handover is a process by which a MN changes its point of attachment to the internet and moves from one network to another. During this process, the MN usually has disconnected from the old network and connects to the new network in transparently manner and with service continuity.

There are two types of handover:

\section{- Horizontal handover}

During this handover process, the $\mathrm{MN}$ changes the point of attachment without affecting the IP layer (L3). Thus the only link layer (L2) participates in the horizontal handover process.

MN moves between different wireless accesses points that are served by the same IP access router (figure 2).

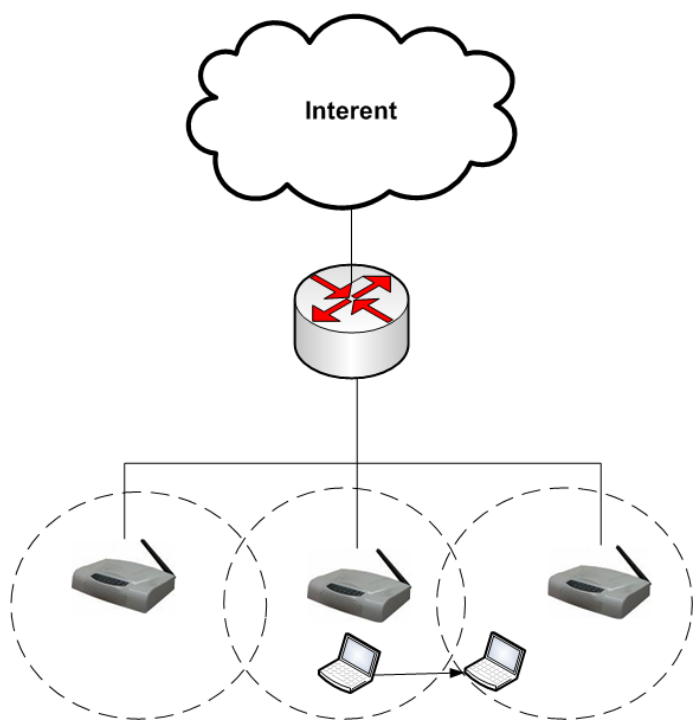

Fig.2 Horizontal handover 
Proc. of the Sixth International Conference on Advances in Computing, Electronics and Communication - ACEC 2017. Copyright (C) Institute of Research Engineers and Doctors. All rights reserved.

ISBN: 978-1-63248-138-2 doi: 10.15224/ 978-1-63248-138-2-12

\section{- Vertical handover}

During this handover process, the MN changes the point of attachment that affects the IP layer (L3). Thus in vertical handover link layer (i.e. L2) as well as IP layer (i.e. L3) participates.

The IP-layer handover occurs during the movement of mobile node connected within different access router (figure 3 ).

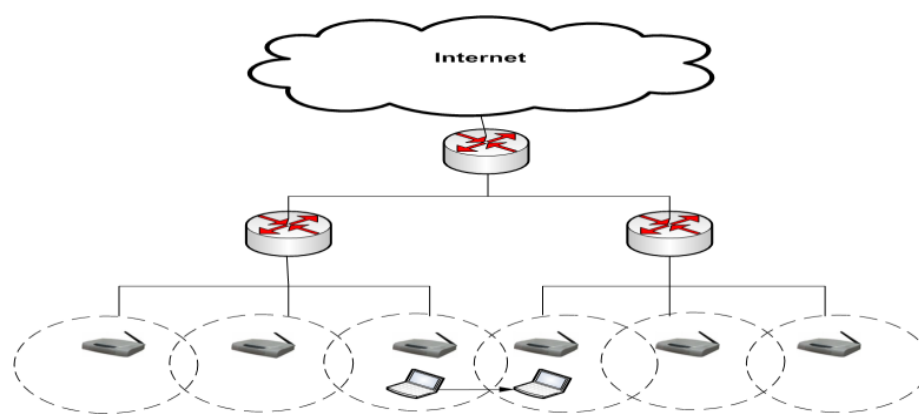

Fig.3 Vertical handover

\section{Handover latency}

In general, the handover latency is defined as the time interval starting from the moment when the Mobile Node leaves the old access medium until it resumes communication with the Correspondent Node at the new access medium [10].

Figure 4 shows handover latency components. T1 is the L2 handover and represents $12 \%$ of the total handover latency. T2 is the time spent by IPv6 to realize that it is attached to a new subnet and to obtain a new IPv6 address (87\%). Finally, MIPv6 operation is carried out in T3 and is composed of the time that the $\mathrm{MN}$ needs to announce its new location (1\%).

Accordingly, the handover delay is given by (1):

$$
\mathrm{T}_{\text {Handover }}=\mathrm{T}_{\mathrm{L} 2 \text { Handover }}+\mathrm{T}_{\mathrm{L} 3 \text { Handover }}
$$

In our paper, we focus on L3 handover delay.

The handover delay in L3 is calculated by the following formula (2).

$$
\mathrm{T}_{\text {L3Handover }}=\mathrm{T}_{\mathrm{IPv} 6}+\mathrm{T}_{\text {MIPv6 }}
$$

Where $\mathrm{T}_{\text {IPv6 }}(3)$ and $\mathrm{T}_{\text {MIPv6 }}(4)$ are:

$$
\begin{aligned}
& \mathrm{T}_{\text {IPv6 }}=\mathrm{T}_{\text {MD }}+\mathrm{T}_{\text {CoA }} \\
& \mathrm{T}_{\text {MIPv6 }}=\mathrm{T}_{\text {HAREG }}+\mathrm{T}_{\text {CNREG }}
\end{aligned}
$$

As Table 1 shows, T2 is the main responsible of the high latency in handover process, so most of the time $(87 \%)$ is devoted to IPv6 tasks.

The parameters presented in the above equations $(1,2,3,4)$ are explained in the following.
TABLE I. HANDOVER DELAY PHASES VS TIME

\begin{tabular}{|c|c|}
\hline Handover phases & Time \\
\hline $\mathrm{T} 1=\mathrm{T}_{\mathrm{L} 2 \text { Handover }}$ & $12 \%$ \\
\hline $\mathrm{T} 2=\mathrm{T}_{\mathrm{IPv} 6}$ & $87 \%$ \\
\hline $\mathrm{T} 3=\mathrm{T}_{\text {MIPv6 }}$ & $1 \%$ \\
\hline
\end{tabular}

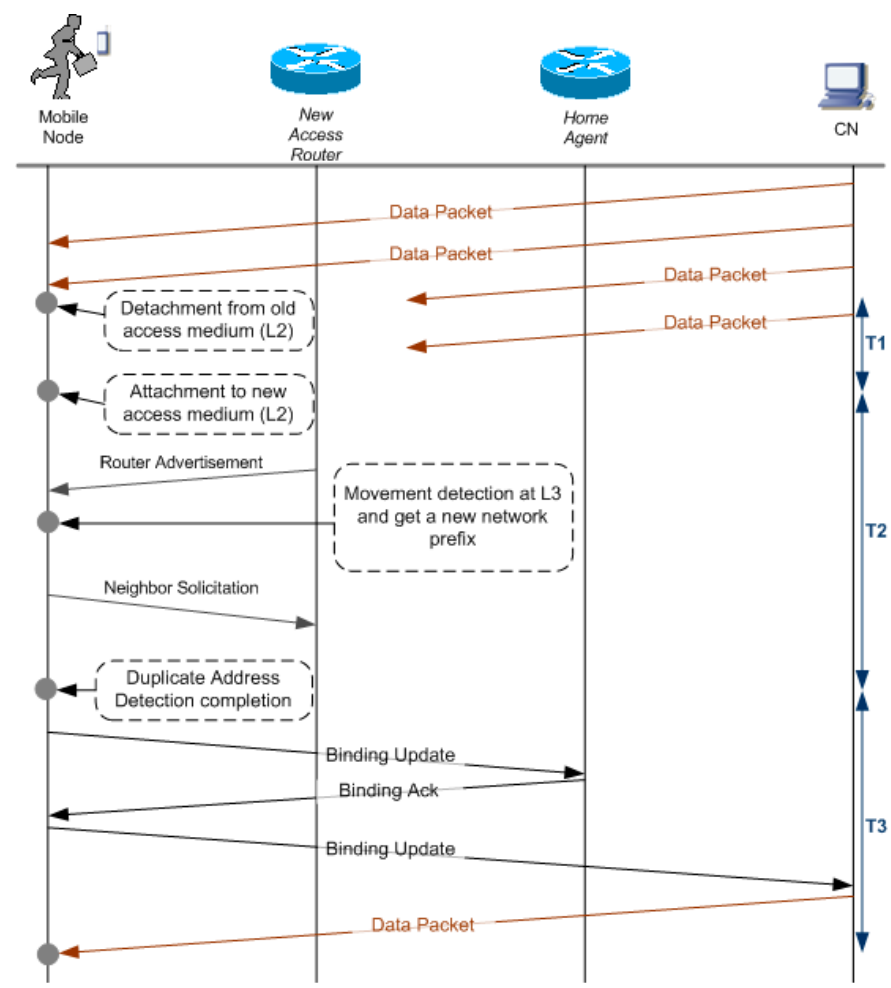

Fig. 4 Handover delay components

A L3 handover procedure can be divided into four phases: Movement Detection, CoA Configuration, Home agent Registration and Route Optimization.

- Movement detection

Movement Detection phase-handover delay $t_{M D}$ is defined as the time interval between the time when the $\mathrm{MN}$ attaches to the new access point (AP) (the finish time of L2 handover and that when the $\mathrm{MN}$ receives the first RA in the new network).

$\mathrm{T}_{\mathrm{MD}}$ is calculated as follows:

$$
\mathrm{t}_{\mathrm{MD}}=\mathrm{t}_{1}-\mathrm{t}_{0}
$$

$t_{1}$ and $t_{0}$ represent the time point of L2 handover's completion and the receiving time of the first RA respectively.

That means: the time between the sending time of the first $\mathrm{RS}$ and the receiving time of the first RA.

- Care of address configuration phase

Handover delay $\mathrm{t}_{\mathrm{CoA}}$ is defined as the time interval between the receiving time of the first $\mathrm{RA}$ after the $\mathrm{MN}$ attached to the 
new network and the sending time of the first first binding update (BU) from MN to HA. It calculated as follows:

$$
\mathrm{T}_{\mathrm{CoA}}=\mathrm{t}_{2}-\mathrm{t}_{1}
$$

Where $t_{2}$ is the first BU sent time.

- Home agent registration phase

Handover delay $t_{R}$ is defined as the delay time between the sending the first $\mathrm{BU}$ from $\mathrm{MN}$ to the $\mathrm{HA}$ and the receiving of the first BA from the HA to MN. It is calculated as follows:

$$
\mathrm{t}_{\mathrm{R}}=\mathrm{t}_{3}-\mathrm{t}_{2}
$$

Where $t_{3}$ is the first $B A$ received time and $t_{2}$ is the first BU sent time.

- Route Optimization phase

The Handover delay $t_{O}$ is the time between the sending of the first $\mathrm{BU}$ from $\mathrm{MN}$ to $\mathrm{CN}$ and the receiving of the first $\mathrm{BA}$ from $\mathrm{CN}$. Is calculated as follows:

$$
\mathrm{t}_{\mathrm{O}}=\mathrm{t}_{4}-\mathrm{t}_{3}
$$

Where $t_{4}$ is the first BA received time from $\mathrm{CN}$. Thus, the total of L3 handover delay is calculated as follows:

$$
\mathrm{T}_{\mathrm{L} 3 \text { Handover }}=\mathrm{t}_{\mathrm{MD}}+\mathrm{t}_{\mathrm{CoA}}+\mathrm{t}_{\mathrm{R}}+\mathrm{t}_{\mathrm{O}}
$$

\section{Router Solicitations and Router Advertisements}

Router Solicitations (RS) and Router Advertisements (RA) help the MN identify that it has changed network and provide it with the necessary information for the creation of the new CoA. While in the standard IPv6, the values for RAs were in the order of 3 to 5 seconds. For the modified Mobile IPv6 these values need to be significantly lower. In this work, we minimize the RS and RA intervals in an effort to deduce their effect on $\mathrm{T}_{\mathrm{MD}}$.

\section{E. Stateless autoconfiguration and Duplicate Address Detection}

The stateless address autoconfiguration mechanism [11] allows a node to generate its own addresses in the following way. The default router advertises prefixe that identify the network associated with a link, while nodes generate an 'interface identifier' that uniquely identifies an interface on each network. A global address is formed by combining the two. The formation of an address must be followed by the DuplicateAddress Detection (DAD) mechanism in order to avoid address duplication on links where stateless address autoconfiguration is used.

Once the MN discovers a new router and creates a new address CoA, it checks its uniqueness within the network. In this paper, we have modified MIPv6 with this feature (DAD) either enabled or disabled.

\section{THE PROPOSED METHOD}

The movement detection phase represents $87 \%$ of handover delay. In our article, we reduce the parameters associated with this phase, namely RA interval, RS interval and DAD process.

\section{A. Reducing Router Advertisement Interval}

Routers send unsolicited RAs to advertise its presence to other nodes in an interval defined by MaxRtrAdvInterval and MinRtrAdvInterval. In our paper, we have modified the default values of these parameters to allow fast movement detection in network layer.

\section{B. Changing the Router Solicitation interval}

In order to reduce $\mathrm{T}_{\mathrm{MD}}$, we have changed the router solicitation intervals and forced the $\mathrm{MN}$ to send a router solicitation message immediately after recognizing that it has moved to a new access point (AP).

\section{Disabling the DAD process}

In our study, we relied on the construction of the identifier from the MAC address; this is why we disable the DAD process. In this way, we reduced the time $t_{D A D}$.

\section{The proposed algorithm}

In this work, we propose an algorithm called Fast Unsolicited Router Advertisement called FURA.

The steps of our algorithm are explained as follows:

- A MN doesn't receive a URA (Unsolicited Router Advertisement). This situation is known because of the absence of a new URA in an interval equal to MaxRtrAdvInterval.

- MN sends a RS cheking the accessibility with the router (in the home network).

- If a RA is not received in a period of time between 0 and MAX_RTR_SOLICITATION_DELAY(1 second), it is possible to suppose that the lost has been caused due to a MN movement

- The MN connects to a new access router, listening RA messages sent by routers periodically. The network prefix received in a RA is employed to configure the new CoA. This address is registered in the Home Agent (HA) and CN. 
Proc. of the Sixth International Conference on Advances in Computing, Electronics and Communication - ACEC 2017. Copyright (C) Institute of Research Engineers and Doctors. All rights reserved.

ISBN: 978-1-63248-138-2 doi: 10.15224/ 978-1-63248-138-2-12

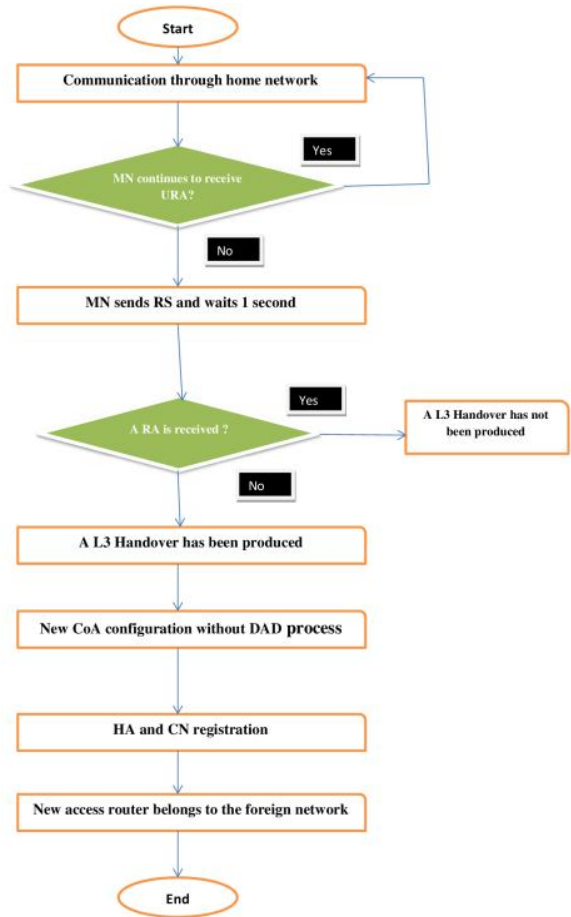

Fig.4.The flowchart of the proposed algorithm FURA

\section{v. SIMULATIONS AND RESULTS}

To evaluate and compare the delay performance of our approach with MIPv6, a simulation technique was accomplished using xMIPv6 module [12]. The simulation environment is shown in Table 1. The network topology used in the simulation is shown in Figure 5. The network is composed of two access routers (AR), two correspondent nodes (CN), two access points (AP) and one mobile node (MN). Each access points is connected to a different access router and configured with a different wireless network name. We assumed that the MN moves from the area of AP1 toward the area of AP2 and then returns to AP1. The handover delay is calculated every time an MN moves in the direction of new access point.

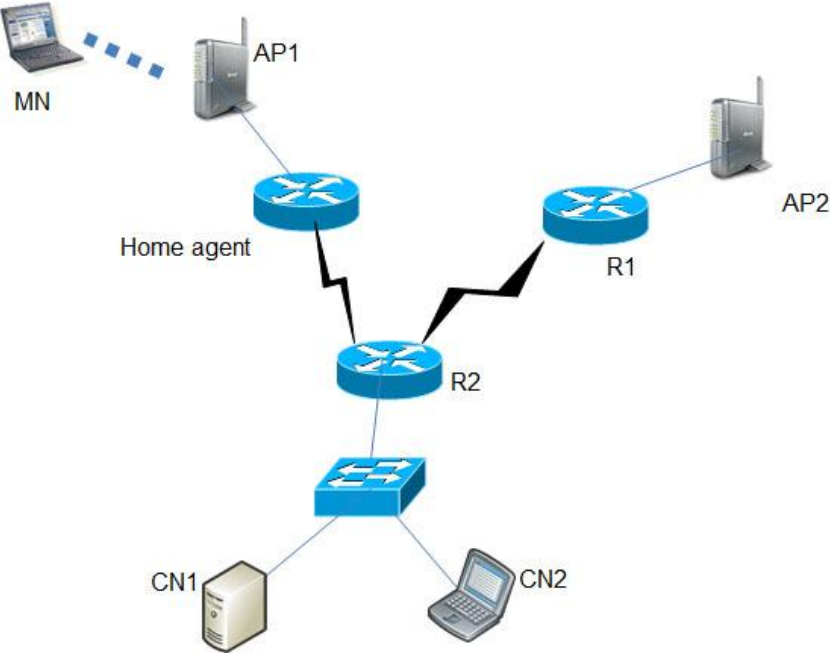

Fig. 5 the network topology used in the Simulation

The two that appear in this section are:

- Impact of unsolicited RA interval, given by MaxRtrAdvInterval and MinRtrAdvInterval.

- Handover delay according to RS interval.

In both cases, our algorithm is compared to Mobile IPv6.

\section{A. Handover delay according to $R A$ interval}

Figure 6 shows L3 handover delay according to Router Advertisement Interval. Obtained data are also shown in Table 2. If routers send unsolicited RAs fast, the time needed to detect the movement is shorter. However, a low configuration of these parameters causes an extra overload in the network.

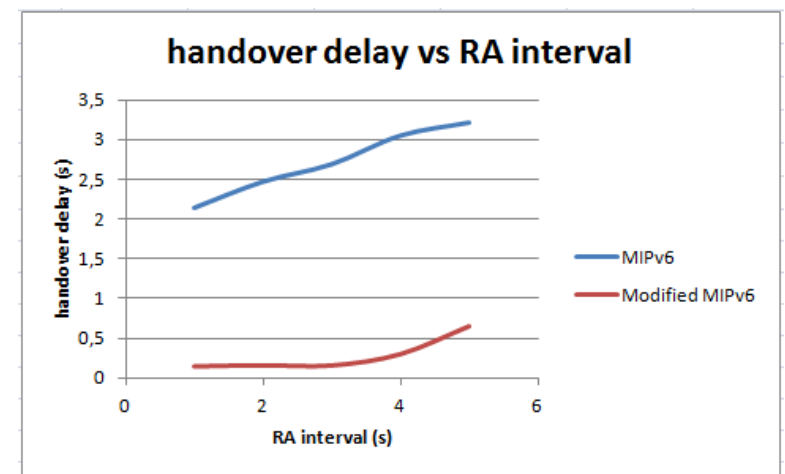

Fig.6. Handover delay according to RA interval

TABLE II. . HANDOVER DELAY IN MIPV6 AND MODIFIED MIPv6

\begin{tabular}{|c|r|r|}
\hline $\begin{array}{l}\text { RA } \\
\text { interval(s) }\end{array}$ & $\begin{array}{l}\text { handover } \\
\text { delay in } \\
\text { MIPv6(s) }\end{array}$ & $\begin{array}{l}\text { handover delay in } \\
\text { Modified MIPv6(s) } \\
\text { (FURA algorithm) }\end{array}$ \\
\hline $0.03-0.07$ & 2,149 & 0.1512074 \\
\hline $0.04-0.08$ & 2,477 & 0.16558 \\
\hline
\end{tabular}


Proc. of the Sixth International Conference on Advances in Computing, Electronics and Communication - ACEC 2017. Copyright (C) Institute of Research Engineers and Doctors. All rights reserved.

ISBN: 978-1-63248-138-2 doi: 10.15224/ 978-1-63248-138-2-12

\begin{tabular}{|r|r|r|}
\hline $0.07-0.11$ & 2,697 & 0.162878 \\
\hline $0.1-0.5$ & 3,055 & 0.30285 \\
\hline $0.5-1.5$ & 3,218 & 0.648477 \\
\hline
\end{tabular}

In Figure 5 we recognize that when we reduce the RA interval, the $\mathrm{T}_{\mathrm{MD}}$ is reduced.

\section{B. Handover delay according to RS interval}

Figure 7 shows the handover delay according to RS interval.

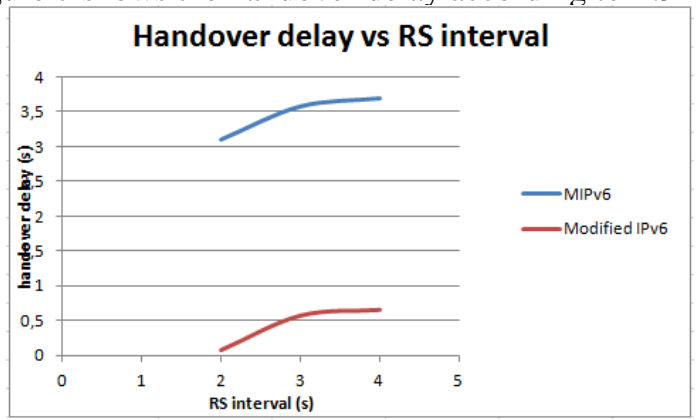

Fig.7 Handover delay according to RS interval

We have disabled DAD process in Mobile IPv6 to minimize the elapsed time in this phase.

From the results, we conclude that our algorithm (FURA) has reduced the time of movement detection and the time of DAD process.

This proof checks the behavior of the proposed FURA algorithm comparing it versus the defined in Mobile IPv6 (RFC6275).

The figure 6 and 7 show the simulation results. When the FURA is used, the handover delay is minimized.

With our approach, if URA interval is low, the handover delay improvement is not so high.

\section{ConCluSION}

MIPv6 is the future generation mobility management protocol. In MIPv6 the handover management is a critical issue. Layer-3 is involved in handover process and responsible for handover delay. Generally, handover is classified as horizontal and vertical handover. Layer-2 is responsible for horizontal handover and Layer-3 is for vertical handover. For MIPv6 eight sub processes are responsible for handover latency. These are movement detection, candidate access router

Discovery, address configuration, duplicate address detection, authentication \& authorization, care-of address registration, Binding Update and binding acknowledgement. In this work we have identified the components responsible for handover latency and we have developed an algorithm called FURA in order to minimize the L3 handover delay.

Regarding the standard Mobile IPv6 handover delay, our numerical results validated by simulations show that our method reduces the L3 handover delay.

Although this paper reduces the handover delay, there are other parameters of delay in MIPv6 handover such as: Binding Update or Binding Acknowledgement, which need to be optimized. The study and improvement of these topics is presented as future work.

\section{REFERENCES}

[1] D. Johnson, C. Perkins, and J. Arkko, "Mobility Support in IPv6," RFC 6275, July 2011. Available from: http://tools.ietf.org/html/rfc6275.

[2] R. Koodli, "Mobile IPv6 fast handovers,", RFC5268,June 2008.

[3] B.Chen,"Reducing Handover latency and improving tcp performance in wireless networks", october 2010.

[4] Georgios Z. Papadopoulos et al.,"Optimizing the handover delay in mobile WSNs",IEEE xplore,21 January 2016.

[5] A Rasem, M St-Hilaire, C Makaya,"Efficient handover with optimized localized routing for Proxy Mobile IPv6",journal of Telcommunication Systems, issue 4/2016.

[6] I.Lakshmi Krishnan, S.Punithavathani,"A Novel Approach to Reduce Handover Latency in Proxy Mobile IPv6 Based on Multi-Homing", July 2016.

[7] P Sharma, H Singh, A Sharma,"Handoff delay Optimization in IEEE 802.16e (Mobile WiMAX) using Fuzzy Expert System",International Journal For Science, Management and Technology (IJSMT), $\operatorname{vol}(7) 2016$.

[8] A.Wei,G.Wei,B.Geller,"Improving Mobile IPv6 Handover in Wireless Network with E-HCF",October 2008.

[9] A.K. Barbudhe,V.K. Barbudhe,C.Dhawale,"Comparison of Mechanisms for Reducing Handover Latency and Packet Loss Problems of Route Optimization in MIPv6",April 2015.

[10] G. Daley, B. Pentland, and R. Nelson, "Movement detection optimizations in Mobile IPv6"," in The 11th IEEE International Conference on Networks (ICON 2003), Sydney, Australia, Sept./Oct.

[11] S. Thomson, T. Narten, T. Jinmei, and H. Soliman: IPv6 Stateless Address Autoconfiguration, RFC4862, September 2007.

[12] E. Kadušić , M. Škrbić , N. Živić , A. Žgalj and A. Hantalašević , "Implementation of a HMIPv6 extension in the INET and xMIPv6 simulation framework with dynamic MAP discovery for OMNeT 4.x", Information and Communication Technology, Electronics and Microelectronics (MIPRO), 2015 38th International Convention on, 16 July 2015 . 